\title{
An Emotion Regulation Transition Model for the Elderly: A Hypothesis
}

\author{
Masa Sasagawa ${ }^{1 *}$, Kate Elliott $^{2}$ and Paul S Amieux ${ }^{2}$ \\ ${ }^{1}$ Bastyr University Research Institute, USA \\ ${ }^{2}$ Bastyr University Research Institute, USA
}

*Corresponding author: Masa Sasagawa, Assistant Research Scientist, Bastyr University Research Institute, 14500 Juanita Dr. NE, Kenmore, WA 98028, USA.

To Cite This Article: Masa Sasagawa, Kate Elliott, Paul S Amieux, An Emotion Regulation Transition Model for the Elderly: A Hypothesis. Am J Biomed Sci \& Res. 2021 - 11(6). AJBSR.MS.ID.001699. DOI: 10.34297/AJBSR.2021.11.001699.

Received: 海 February 02, 2021; Published: 䟧 February 12, 2021

\begin{abstract}
The increasing number of geriatric populations in developed countries poses serious questions about how to promote and sustain independent living and a better quality of life for the elderly, whose physical and mental decline is ultimately unavoidable. Physical and mental decline in the elderly is highly variable and influenced by genetics; environment; socioeconomic status; culture; race; ethnicity; religiosity; family, community, state and national structures; along with many other factors. Physical and cognitive functional decline in the elderly may trigger maladaptive psychosocial behaviors that may include physical isolation and reclusiveness, hostility towards others including professional and family caregivers, depression, suicide and refusal of medication or treatment.

As the elderly population continues to increase in developed countries, primary care and behavioral health workers need additional tools to detect the psychosocial and emotional turmoil that may be associated with this critical transitional period in the elderly, which may occur slowly or may be quite rapid. We propose the development of a tool using less intimidating and potentially robust assessments of elderly in order to identify the risk of this psychosocial and emotional turmoil. After collection of a large dataset on physical and cognitive function, along with an examination of emotional defense mechanism styles, we hypothesize that a respiratory function test combined with analysis of narrative data from a wish-regretadvice conversation may generate a useful functional tool for geriatric populations. The goal of developing an easy screening tool is to reduce care burden for behavioral health workers by promoting and maintaining autonomy and a better quality of life for the elderly to the end.
\end{abstract}

Keywords: Gerontology, Geriatrics, Elderly, Emotional Regulation, Healthy Aging

Abbreviations: FVC: Forced Vital Capacity; PVC: Pulmonary Vital Capacity

\section{Introduction}

Neuroscience research supports the involvement of cognitive control in emotional regulation [1]. Elderly individuals are generally emotionally stable and well-regulated up to age 70-80 years old [2] however, when physical and/or cognitive decline sets in, undesirable emotional states may be concealed by psychosocial and emotional defense mechanisms. For some elderly, perceived mental and physical decline become self-evident earlier than 70 years old due to the impact of changes in life circumstances such as forced retirement, loss of a spouse, changes in the home environment or financial insecurity.
The transition can be rapid, but can also be gradual, slow and insidious. Due to the sensitive nature of the topic of mental/physical decline and impairment in the elderly, who most often wish to remain strong and independent, clinicians and psychologists need to develop tools to detect possible psychosocial and emotional turmoil associated with this critical transitional period that is often fraught with anxiety, depression, worry and fear. Armed with an evidence-supported developmental model of the trajectory of change in emotional processing in the elderly, we may be able to intervene to prevent self-destructive choices and behaviors that include loneliness, depression, suicide, refusal of medication or 
treatment, and refusal of care by family members or health care professionals. The decline of cognitive function in the elderly with its associated defense mechanisms may also make the elderly more vulnerable to fraud and financial exploitation [3].

For the geriatric population, cognitive function tests and batteries of psycho-emotional assessments may appear intimidating if not outright frightening. Elderly patients often acknowledge the reality of physical decline but are more resistant to acknowledging emotional or cognitive decline due to social and personal stigmatization [4]. Recent research has clearly demonstrated that one of the most important health-related functions to maintain in the elderly is pulmonary function [5]. Diminished pulmonary function in older adults often leads to respiratory failure and death. Pulmonary vital capacity (PVC) can be captured during the use of an incentive spirometer that encourages post-operative training of lung capacity during a convalescent period. Furthermore, forced vital capacity (FVC) is associated with total health-related quality of life [6] and peak air flow is a statistically significant indicator in predicting mortality in both genders [7]. If health preventive care guidelines include a routine assessment of respiratory function in elderly populations, public health agencies may be able to detect associated comorbidities, including maladaptive psychosocial and emotional behaviors resulting from a change in emotional regulation.

Another nonthreatening data collection tool that clinicians may use with the elderly is conversation. For instance, by asking elderly patients their current wish-regret-advice list, similar to a bucket list [8-10], clinicians may be able to identify emotional regulation defense mechanisms and other emerging concerns. Two previous studies investigating "bucket lists" among normal aging subjects and "unfinished business" among terminal cancer patients yielded lists of aggregated common themes. Online survey responses were obtained from 3,056 participants.

The mean age and standard deviation of respondents who had a bucket list was 50.0 and 16.0, respectively. The aggregated summary of top categories on the list were:

1. Desire to travel $(78.5 \%)$.

2. Desire to accomplish a personal goal (78.3\%).

3. Desire to achieve specific life milestones (51\%).

4. Desire to spend quality time with friends and family $(16.7 \%)$.

5. Desire to achieve financial stability (16.1\%); and

6. Desire to do a daring activity (15\%).

"Asking patients to list their bucket list is a simple way to elicit their future plans and allows their doctors to help them craft a careplan that will optimize the chances of fruition of the desires listed" (p.5) [10].

In a related study, the presence of unfinished business (UB) and UB-related distress was studied in 161 out of 223 advanced cancer patients, and hopelessness was found to be predictive of UB-related distress [9] and other themes abstracted were:

A. desire for hastened death.

B. anxiety.

C. depression.

D. quality of life issues.

E. spiritual well-being; and

F. purpose/meaning in life.

The study suggested the development of interventions designed to help patients resolve or find solace with UB [9].

Can a respiratory function test and a wish-regret-advice conversation be incorporated into care plans for the elderly who are at risk for psychosocial and emotional turmoil, and before they develop maladaptive behaviors?

\section{Materials and Methods}

In order to build a testable hypothesis based on the above research question, three items or concepts need to be explored:

a. The failure of emotional regulatory mechanisms-risk for emotional turmoil,

b. Assessment of physical health in the elderly, and

c. Cognitive capacity associated with physical health.

We declare that the method of searching existing theories and published articles was not systematic. Available literature searches and discussion among colleagues found the following:

a) Erikson's psychosocial development model and cognitive function [11]

b) Adaptive midlife defense mechanisms proposed by Malone [12]

c) Structural and functional changes in the lung with aging [13]

d) Review of pulmonary function and cognition in aging [14]

e) Three emotional regulatory mechanisms summarized by [2]

Which are:

a) Socioemotional selectivity theory [15].

b) Dynamic integration theory [16] and

c) Life-span theory of control [17]. 
The results section below describes how these concepts were built into a model and a subsequent testable hypothesis.

\section{Results}

We propose a model demonstrating a continuous transition of physical and cognitive decline with aging. The goal for this model is to generate a screening instrument for primary care clinicians or behavioral health teams to identify the risk of maladaptive emotional turmoil among elderly patients. By creating these models, the timing and types of intervention appropriate for preventing the elderly from falling into maladaptive behaviors may be constructed. Emotional maladaptive choices may not be self-evident; however, we hypothesize that stronger effort aimed at emotional regulation is associated with physical and/or cognitive decline, which may be detectable, for example, in conflicting statements found in narrative data, along with pulmonary capacity depression (Figure 1).

\section{Figure $1 \quad$ Aging Transition Model}

Forced/Chosen
physical and
cognitive capacity
commensurate with
socially required
functioning level

Generativity versus

Stagnation: Care
Adaptive Capacity

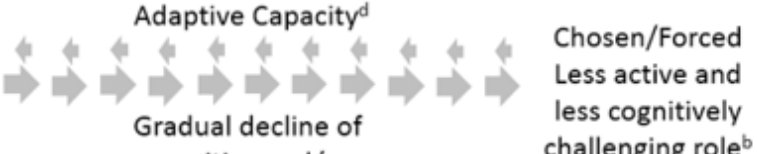

cognitive and/or

physical capacity

with aging ${ }^{c}$

\begin{tabular}{l|l|}
\hline & Legend \\
\hline a & Fully employed and/or physically/mentally functioning elderly \\
\hline b & $\begin{array}{l}\text { After transition, elderly assume a less socially active and less cognitively } \\
\text { challenging role. This state may come by his/her own choice or being forced into it }\end{array}$ \\
c & $\begin{array}{l}\text { All people experience gradual decline in cognitive and physical capacity with aging. } \\
\text { Some transitions may be abrupt due to accidents or life-changing situations }\end{array}$ \\
\hline d & $\begin{array}{l}\text { Each individual adapts to changed situations differently: some are more resilient } \\
\text { and flexible while others are more vulnerable and fragile }\end{array}$
\end{tabular}

Figure 1: Describes an age transition model of physical and cognitive decline (and associated post-Freudian psychosocial stages) that incorporates psychosocial defense mechanisms involved in the aging process surrounding physical or cognitive decline.

Qualitative studies yield extremely valuable yet very diverse datasets which are highly dependent on the method and the interpretive lens [18]. We anticipate that the interpretation of the Table 1.

\begin{tabular}{|c|c|}
\hline Emotional Regulatory Mechanism of Elderly & Description [2] \\
\hline Socioemotional Selectivity Theory & $\begin{array}{l}\text { Holds that anticipated endings such as the sense that life is running out give primacy to enhanc- } \\
\text { ing emotionally gratifying experiences in the moment as opposed to maximizing future rewards. }\end{array}$ \\
\hline Dynamic Integration Theory & $\begin{array}{l}\text { Diminishing cognitive capacities associated with age make it more difficult to integrate and ac- } \\
\text { cept negative feelings, and therefore older adults favor affect optimization over affect complexity }\end{array}$ \\
\hline Life-span Theory of Control & $\begin{array}{l}\text { An individual's capacity to control their environment and achieve their developmental goals } \\
\text { declines in older adulthood }\end{array}$ \\
\hline
\end{tabular}

The suggested theories of emotional regulation described by [2] are:
a) Socioemotional selectivity theory [15]
b) Dynamic integration theory [16] and
c) life-span theory of control [17].

The interpretive method of this directed content analysis [19] can be performed by defining codes from each theory and utilizing a tool originally developed for marketing research analysis called the affinity analysis [20] (Table 1). narrative conversational data will be optimized by incorporating existing theories of emotional regulation mechanisms used by the elderly (Table 1).

\section{Ego Integration versus ,} Despair: Wisdom 
order to abstract the state of mind, psychological vulnerability, and behavioral choices, hence identifying the risk of emotional turmoil. A quantification of the level of risk needs to be developed for operationalization of the model. Furthermore, in order to validate the model, a variety of measurements must be taken, including cognitive function tests, physical function measurements (frailty index, cardiovascular fitness), mood states and daily functioning (sleeping, eating, socializing). Data from diverse sources can then be used for correlation or pathway analyses. Respiratory functions are quantifiable.

If the model can be validated, practitioners and behavioral health teams will then need just two types of data:

1. The respiratory capacity test; and

2. The wish-regret-advice narrative data (Appendix).

No extensive cognitive tests or batteries of psychosocial assessments are needed.

\section{Discussion}

When we consider interventions that can be designed to prevent emotional turmoil in the elderly as a result of cognitive and physical decline, interventions that help maintain physical and cognitive capacities as much as possible and restore weakened systems would of course be optimal. In one theory of resiliency [21], some individuals are able to maintain their emotional and cognitive functionality despite facing high level of stress and an adverse environment, and these individuals may possess psychosocial defense mechanisms that provide support and resiliency during stressful periods and environments. Psychosocial defense mechanisms can be both internal and external and may also contribute to resiliency. Peer support groups, partnerships, financial supports, family members, neighbors, friends, community, spiritual congregations, etc., represent external resources that can support resiliency, whereas higher education, psychological hardiness, high internal locus of control, proper attachment style, and certain personality characteristics may be internal mechanisms of psychosocial resiliency.

\section{Limitations}

Besides the lack of supportive data, the limitations of this model are:

1. It requires a pragmatic protocol-obtaining accurate data during this transition process in the elderly is challenging and requires innovative research methodologies, such as the involvement of different academic disciplines (epidemiology, psychology, sociology, and nursing science) that can perform coding on the interview narrative data from multiple perspectives and lenses; and
2. The divergent dimensionality of personality factorsexpression of emotional turmoil and subsequent defense mechanisms are highly individualistic and are of course influenced by place, culture, socioeconomic status, race, ethnicity, religion, family and community structure, environment and many other factors. Thus, creating a robust model will require a large dataset from diverse cultural and social contexts.

\section{Conclusion}

The purpose of identifying risk and preventing subsequent maladaptive behavior change is to improve self-care capacity in elderly people. The goal of this model is not to impose more burden of care on healthcare systems, but to provide new tools to identify and intervene effectively before maladaptive behavior develops in the elderly. Regardless of the age and capacity found in the elderly, service providers, including healthcare workers, should focus on enhancing the self-reliance and independence of elderly clients or patients so that they may enjoy more freedom and a higher quality of life to the end.

\section{Author Contribution}

All authors contributed to the discussion, drafting and revising of the article; gave final approval of the version to be published; and agree to be accountable for all aspects of the work.

\section{Conflict of Interest and Funding}

Authors received no grant funding. No competing interest is declared by the authors.

\section{References}

1. Ochsner KN, Gross JJ (2005) The cognitive control of emotion. Trends in Cognitive Sciences 9(5): 242-249.

2. Scheibe S, Carstensen L (2010) Emotional aging: Recent findings and future trends. Journals of Gerontology-Series B Psychological Sciences and Social Sciences 65B(2): 135-144.

3. Deliema M, Deevy M, Lusardi A, Mitchell OS (2018) Financial fraud among older Americans: Evidence and implications. J Gerontol B Psychol Sci Soc Sci 75(4): 861-868.

4. Raue PJ, Meyers BS (1997) An overview of mental health services for the elderly. New Directions for Mental Health Services (76): 3-12.

5. Pierobon A, Bottelli ES, Ranzini L, Bruschi C, Maestri R, et al. (2017) COPD patients' self-reported adherence, psychosocial factors and mild cognitive impairment in pulmonary rehabilitation. International Journal of COPD 12: 2059-2067.

6. Wen Y, Wang D, Zhou M, Zhou Y, Guo Y, et al. (2019) Potential effects of lung function reduction on health-related quality of life. International Journal of Environmental Research and Public Health 16(2).

7. Eekhoff EM W, Van Schoor NM, Biedermann JS, Oosterwerff MM, De Jongh R, et al. (2019) Relative importance of four functional measures as predictors of 15-year mortality in the older Dutch population. BMC Geriatr 19(1): 1-10.

8. Bohiigian G (2016) Someday I' ll Complete My Bucket List. Missouri Medicine 113: 345 
9. Masterson M, Slivjak E, Jankauskaite G, Breitbart W, Pessin H, et al. (2018). Beyond the bucket list: Unfinished and business among advanced cancer patients. Psychooncology 27(11):2573-2580.

10. Periyakoil VS, Neri E, Kraemer H (2018) Common Items on a Bucket List. Journal of Palliative Medicine 21(5): 652-658.

11. Malone J, Liu S, Vaillant G, Rentz D, Waldinger R, et al. (2016) Midlife Eriksonian Psychosocial Development: Setting the Stage for Cognitive and Emotional Health in Late Life. Dev Psychol 52(3): 496-508.

12. Malone J, Cohen S, Liu S, Vaillant G, Waldinger R, et al. (2013) Adaptive midlife defense mechanisms and late-life health. Personality and Individual Differences 55(2): 85-89.

13. Lowery EM, Brubaker A L, Kuhlmann E, Kovacs EJ (2013) The aging lung. Clinical Interventions in Aging 8: 1489-1496.

14. Duggan E, Graham R, Piccinin A, Jenkins N, Clouston S, et al. (2020) Systematic Review of Pulmonary Function and Cognition in Aging. J Gerontol B Psychol Sci Soc Sci 75(5): 937-952.
15. Carstensen L, Turan B, Scheibe S, Ram N, Ersner-Hershfield H, et al. (2011) Emotional Experience Improves with Age: Evidence Based on Over 10 Years of Experience Sampling. Psychol Aging 26(1): 21-33.

16. Labouvie-Vief G, Lumley MA, Jain E, Heinze H (2003) Age and gender differences in cardiac reactivity and subjective emotion responses to emotional autobiographical memories. Emotio 3(2): 115-126.

17. Heckhausen J, Schulz R (1995) A life-span theory of control. Psychological Review 102(2): 284-304.

18. Creswell J (2007) Qualitative Inquiry \& Research Design: Choosing Among Five Approaches ( $2^{\text {nd }}$ edn.). Thousand Oaks, CA: Sage Publication, Inc. American Psychological Association.

19. Hsieh HF, Shannon SE (2005) Three approaches to qualitative content analysis. Qualitative Health Research, 15(9): 1277-1288.

20. IBM Corp (2012) IBM SPSS Modeler Professional p. 1-8.

21. Richardson, GE (2002) The metatheory of resilience and resiliency. Journal of Clinical Psychology 58(3): 307-321. 